\title{
Sequential Bayesian technique: An alternative approach for software reliability estimation
}

\author{
S CHATTERJEE* $^{*}$ S S ALAM ${ }^{\dagger}$ and R B MISRA ${ }^{\ddagger}$ \\ *Department of Applied Mathematics, Indian School of Mines University, \\ Dhanbad 826004 \\ 'Department of Mathematics, Indian Institute of Technology, Kharagpur 721302 \\ Reliability Engineering Centre, Indian Institute of Technology, \\ Kharagpur 721302 \\ e-mail: chatterjee_subhashis@ rediffmail.com; alam@maths.iitkgp.ernet.in; \\ ravi@ee.iitkgp.ernet.in
}

MS received 8 October 2007; revised 15 July 2008

\begin{abstract}
This paper proposes a sequential Bayesian approach similar to Kalman filter for estimating reliability growth or decay of software. The main advantage of proposed method is that it shows the variation of the parameter over a time, as new failure data become available. The usefulness of the method is demonstrated with some real life data.
\end{abstract}

Keywords. Software reliability; Bayesian sequential estimation; Kalman filter.

\section{Introduction}

As computers are used in various fields of life including business and safety critical systems, software faults have become the major factor that causes critical problems. Hence, there exists an increasing demand for highly reliable software. Software reliability models provide quantitative measures of the reliability of a software system during its development phase. Research activities in the field of software reliability have been conducted since early seventies. Detail studies about software reliability are given in (Xie 1991, Musa et al 1987, Shooman 1968). Some important software reliability growth models (Jelenski \& Moranda 1972, Shooman 1972, Schick \& Wolverton 1978, Musa 1975, Littlewood \& Verrall 1973, Xie 1987, Goel \& Okumoto 1979, Singpurwalla \& Soyer 1985, Yamada et al 1983, Yamada et al 1984, Yamada et al 1986, Yamada et al 1993) have been developed considering perfect debugging and immediate error removal. Incorporating some realistic issues like imperfect debugging and learning process of software developers some other important software reliability growth models (Chatterjee et al 1997, Sumita \& Shantikumar 1986, Fakhre-Zakeri \& Slud 1995, Zeephongsekul et al 1994, Xie et al 1993, Pham 1996, Chatterjee et al 1998, Gokhale et al 2006, Dai et al 2005, Xie et al 2004, Chatterjee et al 2004, Park \& Lee 2003) have also been developed.

Software undergoes several stages of testing before it is put into operation. In every stage of testing, modification and correction are made with the hope of increasing reliability. It is 
very important to know, whether a particular modification or series of modifications lead to the growth of reliability, so that a software engineer can decide when to stop the process of testing. As errors are removed from the software the time between failures gradually increases. With the knowledge of time between failures, it is important to know

(i) whether the modifications made in software are beneficial,

(ii) whether the modifications lead to overall growth or decay of reliability, and

(iii) about next time between failures.

Considering these points researchers have proposed software reliability models using autoregressive process (Singpurwalla \& Soyer 1985).

In this paper, sequential Bayesian estimation procedure (Soman \& Misra 1993) is used for estimating reliability of software. Several regression models like forward section, backward eliminations, step-wise and all sub-set regressions are available in the literature. All these techniques are one shot or batch processing in nature, since the model parameter estimates are calculated are based on the entire data set. One has to repeat the whole process again if new data set is added to the old data set or to get a new estimate. This is computationally undesirable. The sequential estimation technique described here to accomplish this task is more efficient than the available regression techniques. A simple power law model has been used here. Application of the proposed technique has been illustrated with real life data for model validation.

\section{General sequential estimation procedures}

A sequential maximum a posteriori estimation procedure based on Bayesian approach is discussed here. The procedure is capable of utilizing the prior information. Let the general regression model be

$$
Y=B_{0}+\sum_{j=1}^{q-1} B_{j} X_{j}+\varepsilon .
$$

The equation for the Bayesian estimation of the model parameters, $\hat{B}$, is given as

$$
\hat{B}=M+P X^{T} Q^{-1}(Y-X M),
$$

where $P$ is the covariance matrix of estimators $(q \times q)$, given as

$$
P=\left(X^{T} Q X^{-1}+V^{-1}\right) .
$$

$\hat{B}$ : estimated parameter vector $(q \times 1)$

$M:$ mean value of parameter vector $(q \times 1)$ known from the prior information

$X$ : independent variable matrix $(n \times 1)$

$V$ : covariance matrix of $B$ known from prior information

$Q$ : covariance matrix of errors.

Substituting $B_{i}=B_{i+1}, M=M_{i}, Y=Y_{i+1}, P=P_{i+1}, V=P_{i,} X=X_{i+1}$ and $Q=C_{i+1}$ we get the recursive form of equation (2) and (3). Here $C$ is a $m \times m$ diagonal covariance 
matrix of error and $\mathrm{m}$ is the number of observations. Substituting the above expressions in equation (2) and (3) we get

$$
B_{i+1}=B_{i}+P_{i+1} X_{i+1}^{T} C_{i+1}\left(Y_{I+1}-X_{i+1} B_{i}\right)
$$

and

$$
P_{i+1}=\left(X_{i+1}^{T} C_{i+1}^{-1} X_{i+1}+P_{i}^{-1}\right)^{-1}
$$

From matrix inversion theorem we know that,

$$
(A+B C D)^{-1}=A^{-1}-A^{-1} B\left(C^{-1}+D A^{-1} B\right)^{-1} D A^{-1} .
$$

Hence equation (5) may be written as follows

$$
P_{i+1}=P_{i}-P_{i} X_{i+1}^{T}\left(X_{i+1} P_{i} X_{i+1}^{T}+C_{i+1}\right) .
$$

Let $R=P_{i} X_{i+1}^{T} C_{i+1} \& H=X_{i+1}$. Then the following matrix identity holds

$$
(I+R H)^{-1} R=R(I+H R)^{-1} .
$$

Therefore, substituting the values of $\mathrm{R}$ and $\mathrm{H}$ we get,

$$
\begin{aligned}
\left(I+P_{i} X_{i+1}^{T} C_{i+1} X_{i+1}\right)^{-1} P_{i} X_{i+1}^{T} C_{i+1} & =P_{i} X_{i+1}^{T} C_{i+1}^{-1}\left(I+X_{i+1} P_{i} X_{i+1}^{T} C_{i+1}^{-1}\right)^{-1} \\
\left(X_{i+1}^{T} C_{i+1} X_{i+1}+P_{i}^{-1}\right)^{-1} P_{i} X_{i+1}^{T} C_{i+1} & =P_{i} X_{i+1}^{T} C_{i+1}^{-1}\left(I+X_{i+1} P_{i} X_{i+1}^{T} C_{i+1}^{-1}\right)^{-1} \\
\left(X_{i+1}^{T} C_{i+1}^{-1} X_{i+1}+P_{i}\right)^{-1} P_{i}^{-1} X_{i+1}^{T} C_{i+1}^{-1} & =P_{i} X_{i+1}^{T} C_{i+1}^{-1} C_{i+1}\left(C_{i+1}+X_{i+1} P_{i} X_{i+1}^{T}\right)^{-1} \\
P_{i+1} X_{i+1}^{T} C_{i+1}^{-1} & =P_{i} X_{i+1}^{T}\left(X_{i+1} P_{i} X_{i+1}^{T}+C_{i+1}^{-1}\right)^{-1} .
\end{aligned}
$$

Substituting equations (6) \& (7) in (4) \& (5) we get,

$$
\begin{aligned}
& A_{i+1}=P_{i} X_{i+1}^{T} \\
& D_{i+1}=C_{i+1}+X_{i+1} A_{i+1} \\
& K_{i+1}=A_{i+1} D_{i+1} \\
& E_{i+1}=Y_{i+1}-X_{i+1} B_{i} \\
& B_{i+1}=B_{i}+K_{i+1} E_{i+1} \\
& P_{i+1}=P_{i}-K_{i+1} A_{i+1}^{T} .
\end{aligned}
$$

Equations (8) to (13) are the governing equations for the sequential estimation procedure of the parameters. If the number of observations is one then no matrix inversion is involved and the computation becomes efficient. Thus for one observation, equation (8) to (13) may be rewritten as follows; 


$$
\begin{aligned}
A_{i+1} & =\sum P_{u k, i} X_{k, i+1} \\
D_{i+1} & =\sigma_{i+1}^{2}+\sum X_{k, i+1} A_{k, i+1} \\
K_{u, i+1} & =\frac{A_{u, i+1}}{D_{i+1}} \\
E_{i+1} & =\left(Y_{i+1}-\sum X_{k, i+1} B_{k, i}\right) \\
B_{u, i+1} & =B_{u, i}+K_{u, i+1} E_{i+1} \\
P_{u v, i+1} & =P_{u v, i}-K_{u, i+1} A_{v, i+1},
\end{aligned}
$$

where $u=1,2,3, \ldots, q, v=1,2,3, \ldots, q, q$ is the number of parameters and $\sigma_{i+1}^{2}$ is the variance of $Y_{i+1}$. Here in equation (15) $S$ is used instead of $\sigma_{i+1}^{2}$ to denote the error variance obtained from linear regression method. So equation (15) becomes

$$
D_{i+1}=S+\sum X_{k, i+1} A_{k, i+1} \text {. }
$$

\section{Estimation of model parameters}

In the following paragraphs the description of the model is followed by the parameter estimation using proposed algorithm.

Let $X_{t}=X_{t-1}^{\theta} \delta$ where $\theta$ is constant and values of $\theta>1$ means growth of reliability and $\theta<1$ means decay of reliability. $\delta$ is the error due to some uncertainty in power law. Taking natural logarithm on both sides we get

$$
\begin{aligned}
\log X_{t} & =\theta \log X_{t-1}+\log \delta \\
\text { or } Y_{t} & =\theta Y_{t-1}+B_{1},
\end{aligned}
$$

where $Y_{t}=\log X_{t}, B_{1}=\log \delta$.

To apply the above-mentioned algorithm for general sequential procedure given in equations (14) to (19) the expression (21) becomes

$$
Y=B_{1} X_{1}+B_{2} X_{2}
$$

where $Y$ is $Y_{t}, B_{1}=\log \delta, B_{2}=\theta, X_{2}=Y_{t-1}$ and $X_{1}$ is a dummy variable taking a constant value 1 . Here $t$ denotes the stage of testing and $X_{t}$ denotes the time between failures. For illustration purpose System 40 data (Musa 1979) is used. The value of $Y_{t}$ i.e. the original failure data is given in table 1 and the estimated value of $B_{1}$ and $B_{2}$ are given in table 2 for each stage of testing $t$. Figure 1 shows the variation of $B_{2}$ i.e. $\theta$ with $t$.

\section{Conclusion}

A Bayesian sequential estimation procedure for estimating software reliability is developed and illustrated with System 40 data of (Musa 1979). The main advantage of this method over others is, it can use the prior estimates of the parameters and can show the variation of parameters over a time as new failure data are available. The objective of the work is to 
Table 1. Value of $Y_{t}$ corresponding to stage of testing $t$.

\begin{tabular}{|c|c|c|c|c|c|c|c|c|c|}
\hline$t$ & $Y_{t}$ & $t$ & $Y_{t}$ & $t$ & $Y_{t}$ & $t$ & $Y_{t}$ & $t$ & $Y_{t}$ \\
\hline 1 & $9 \cdot 574$ & 22 & 8.046 & 43 & $4 \cdot 700$ & 64 & $9 \cdot 574$ & 85 & $12 \cdot 720$ \\
\hline 2 & $9 \cdot 104$ & 23 & $10 \cdot 845$ & 44 & $10 \cdot 002$ & 65 & $10 \cdot 450$ & 86 & 14.199 \\
\hline 3 & 7.965 & 24 & 9.741 & 45 & 11.012 & 66 & $10 \cdot 586$ & 87 & $11 \cdot 370$ \\
\hline 4 & 8.648 & 25 & $7 \cdot 544$ & 46 & $10 \cdot 862$ & 67 & $12 \cdot 720$ & 88 & $12 \cdot 202$ \\
\hline 5 & 9.989 & 26 & $8 \cdot 594$ & 47 & 9.437 & 68 & $12 \cdot 598$ & 89 & $12 \cdot 279$ \\
\hline 6 & $10 \cdot 196$ & 27 & 11.039 & 48 & $6 \cdot 664$ & 69 & $12 \cdot 086$ & 90 & $11 \cdot 366$ \\
\hline 7 & 11.639 & 28 & $10 \cdot 119$ & 49 & $9 \cdot 229$ & 70 & $12 \cdot 276$ & 91 & $11 \cdot 366$ \\
\hline 8 & $11 \cdot 627$ & 29 & $10 \cdot 178$ & 50 & $8 \cdot 967$ & 71 & 11.960 & 92 & 14.411 \\
\hline 9 & $6 \cdot 492$ & 30 & 5.894 & 51 & $10 \cdot 353$ & 72 & $12 \cdot 024$ & 93 & $8 \cdot 333$ \\
\hline 10 & $7 \cdot 901$ & 31 & $9 \cdot 546$ & 52 & $10 \cdot 987$ & 73 & 9.287 & 94 & $8 \cdot 07$ \\
\hline 11 & $10 \cdot 267$ & 32 & $9 \cdot 619$ & 53 & $12 \cdot 607$ & 74 & 12.495 & 95 & $12 \cdot 202$ \\
\hline 12 & $7 \cdot 683$ & 33 & $10 \cdot 385$ & 54 & $7 \cdot 154$ & 75 & 14.556 & 96 & $12 \cdot 783$ \\
\hline 13 & 8.89 & 34 & $10 \cdot 636$ & 55 & 10.003 & 76 & $13 \cdot 327$ & 97 & $13 \cdot 258$ \\
\hline 14 & 11.59 & 35 & $8 \cdot 333$ & 56 & $9 \cdot 86$ & 77 & 8.946 & 98 & 12.753 \\
\hline 15 & $8 \cdot 349$ & 36 & $11 \cdot 314$ & 57 & $7 \cdot 86$ & 78 & $14 \cdot 782$ & 99 & $10 \cdot 353$ \\
\hline 16 & 9.043 & 37 & 9.487 & 58 & $10 \cdot 575$ & 79 & 14.896 & 100 & 12.489 \\
\hline 17 & 9.602 & 38 & 8.139 & 59 & 10.929 & 80 & $12 \cdot 139$ & & \\
\hline 18 & $9 \cdot 379$ & 39 & $8 \cdot 671$ & 60 & $10 \cdot 660$ & 81 & 9.798 & & \\
\hline 19 & 8.586 & 40 & 6.461 & 61 & 12.497 & 82 & 12.09 & & \\
\hline 20 & 8.787 & 41 & $6 \cdot 461$ & 62 & $11 \cdot 374$ & 83 & $11 \cdot 382$ & & \\
\hline 21 & 8.779 & 42 & 7.965 & 63 & 11.915 & 84 & $13 \cdot 367$ & & \\
\hline
\end{tabular}

Table 2. Estimated values of $\delta$ and $\theta$ corresponding to each stage of testing $t$.

\begin{tabular}{|c|c|c|c|c|c|c|c|c|c|c|c|c|c|c|}
\hline$t$ & $\delta$ & $\theta$ & $t$ & $\delta$ & $\theta$ & $t$ & $\delta$ & $\theta$ & $t$ & $\delta$ & $\theta$ & $t$ & $\delta$ & $\theta$ \\
\hline 1 & & & & & & 43 & & & 4 & & & 85 & 02 & 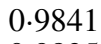 \\
\hline 2 & 206 & 586 & 23 & & & 44 & 0182 & 9786 & 65 & & 9805 & 8 & 403 & 835 \\
\hline 3 & 74 & 1.043 & 24 & $1 \cdot 0164$ & 1.0018 & 45 & 1.0244 & 841 & 66 & 22 & 820 & 7 & 401 & 857 \\
\hline 4 & & & 25 & & & 46 & & & 67 & & & 0 & & \\
\hline 5 & $1 \cdot 0145$ & $1 \cdot 0285$ & 26 & 143 & 892 & 47 & $1 \cdot 0248$ & 0.9867 & 68 & 1 . & & 89 & 12 & 0.9828 \\
\hline 6 & 1.0 & $1 \cdot($ & 27 & 1 . & 0.99 & 48 & 1 . & 0 . & 69 & 1 & 2 & 90 & 2 & 31 \\
\hline 7 & & & 28 & & & 49 & & & 7 & & & 1 & & \\
\hline 8 & & & 29 & & & 50 & & & 71 & & & 32 & & \\
\hline 9 & & & 30 & & & 51 & & & 72 & 1 . & & 93 & & \\
\hline 10 & 1.0096 & 0.9 & 31 & & & 52 & & 0.9 & 73 & & & 94 & & 0.9776 \\
\hline 11 & $1 \cdot 0118$ & 0.9 & 32 & $1 \cdot($ & & 53 & & & 74 & & & J & & 76 \\
\hline 12 & & & 33 & & & 54 & & & 75 & & & 96 & & 0.9806 \\
\hline 13 & 1.0119 & 0.9946 & 34 & $1 \cdot 01$ & & 55 & & & 76 & & & 97 & & \\
\hline 14 & & $1 \cdot 0005$ & 35 & & & 56 & & & 77 & & & 0 & & \\
\hline 15 & 1.0167 & $1 \cdot 0144$ & 36 & $1 \cdot 0$ & 0.5 & 57 & 1 . & 0. & 78 & $1 \cdot($ & 0 & 99 & 72 & 0.9818 \\
\hline 16 & $1 \cdot 0137$ & 0.9928 & 37 & 1.0207 & 0.9935 & 58 & 1.0294 & 0.9771 & 79 & & 0.9867 & 100 & & 0.9793 \\
\hline 17 & 1.0145 & 0.996 & 38 & 1.0202 & 0.9882 & 59 & 1.0312 & 0.9810 & 80 & 1.0378 & 0.9872 & 101 & 1.0472 & 0.9814 \\
\hline 18 & $1 \cdot 0151$ & 0.9987 & 39 & 1.019 & & 60 & 1.03 & & 81 & 1.0392 & & & & \\
\hline 19 & 1.0149 & 0.9976 & 40 & $1 \cdot 02$ & & 61 & 1.0312 & 0.98 & 82 & 1.0393 & 0.9795 & & & \\
\hline 20 & 1.0142 & 0.9943 & 41 & $1 \cdot 018$ & 0.9819 & 62 & $1 \cdot 0312$ & 0.9854 & 83 & $1 \cdot 0401$ & 0.9822 & & & \\
\hline 21 & 1.0144 & 0.9951 & 42 & 1.0189 & 0.9821 & 63 & 1.0317 & 0.9834 & 84 & 1.0401 & 0.9815 & & & \\
\hline
\end{tabular}




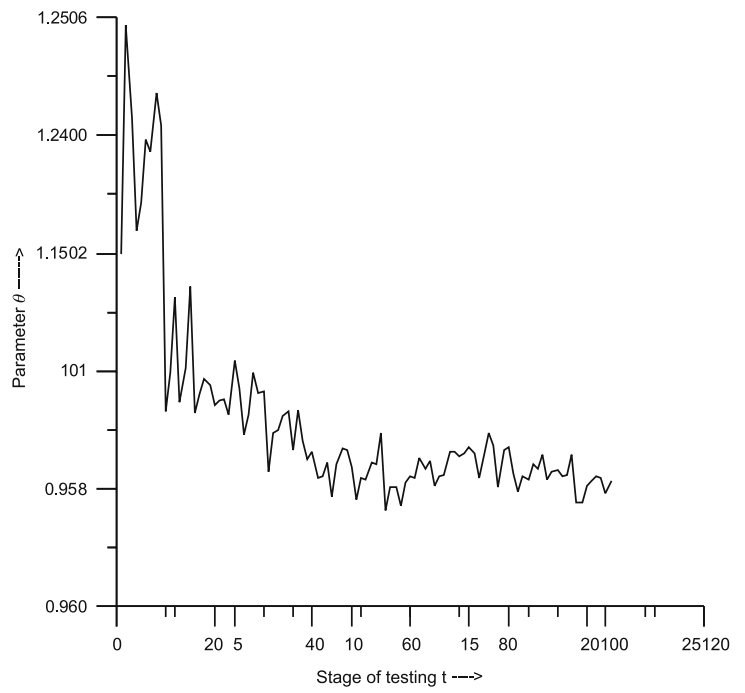

Figure 1. Variation of $\theta$ w.r.t. stage of testing $t$.

establish an alternative simplified approach for estimating reliability of software. The proposed method will be computationally very simple and efficient for software engineers. One can use this technique at any stage of testing.

Author acknowledges University Grants Communication (UGC), New Delhi for financial help to Dr S Chatterjee in the project number F.No.33-115/2007(SR).

\section{References}

Chatterjee S, Misra R B, Alam S S 1997 Joint effect of test effort and learning factor on software reliability and optimal release policy. Int. J. Sys. Sci. 28(4): 391-396

Chatterjee S, Misra R B, Alam S S 1998 A generalized shock model for software reliability. Comput. Elect. Eng.-An Int. J. 24: 363-368

Chatterjee S, Misra R B, Alam S S $2004 \mathrm{~N}$-version programming with imperfect debugging. Comput. Elect. Eng. 30(6): 453-463

Dai Y S, Xie M, Poh K L 2005 Modelling and analysis of correlated software failures of multiple types. IEEE Trans. Rel. 54(1): 100-106

Fakhre-Zakeri I, Slud E 1995 Mixture models for reliability of software with imperfect debugging: Identifiably of parameters. IEEE Trans. Rel. 44: 104-113

Goel A L, Okumoto K 1979 A time-dependent error detection rate model for software reliability and other performance measure. IEEE Trans. Rel. R-28: 206-211

Gokhale S S, Lyu M R, Trivedi K S 2006 Incorporating fault debugging activities into software reliability models: A simulation approach. IEEE Trans. Rel. 55(2): 281-292

Jelinski Z, Moranda P B 1972 Software reliability research statistical computer performance evaluation. W Freiberger, Ed. Academic, NY, 465-484

Littlewood B, Verrall J L 1973 A bayesian reliability growth model for computer software. Appl. Statist. 22: 332-346

Musa J D 1975 A theory of software reliability and its application. IEEE Trans. Software Eng. SE-1: $312-327$ 
Musa J D 1979 Software reliability data, New York: DACS, RADC/ISISI

Musa J D, Iannino A, Okumoto K 1987 Software reliability measurement. Prediction, Application, McGraw-Hill Int. Ed.

Park D H, Lee C H 2003 Markovian imperfect software debugging model and its performance measures. Stochastic Analysis And Applications 21(4): 849-864

Pham H 1996 A software cost model with imperfect debugging random life cycle and penalty cost. Int. J. Sys. Sci. 27: 455-463

Schick G J, Wolverton R W 1978 An analysis of competing software reliability model. IEEE Trans. Software Eng. SE-4: 104-120

Shooman M L 1968 Probabilistic reliability: An engineering approach. (NY: McGraw Hill)

Shooman M L 1972 Probabilistic models for software reliability prediction. Statistical Computer Performance Evaluation, W Freiberger, Ed. Academic, NY, 485-502

Singpurwalla N D, Soyer R 1985 Assessing (Software) reliability growth using a random co-efficient autoregressive process and its ramifications. IEEE Trans. Software Eng. SE-11: 1456-1464

Soman K P, Misra K B 1993 On Bayesian estimation of system reliability. Microelectronic Reliability 33: $1455-1459$

Sumita U, Shantikumar J G 1986 A software reliability model with multiple-error introduction \& removal. IEEE Trans. Rel. R-35: 459-462

Xie M 1987 A shock model for software reliability. Microelectronic Reliability 27: 717-724

Xie M 1991 Software reliability modelling. World Scientific Press

Xia G, Zeephongsekul P, Kumar S 1993 Optimal software release policy with a learning factor for imperfect debugging. Microelectronic Reliability 33: 81-86

Xie M, Dai Y S, Poh K L 2004 Distributed system availability in the case of imperfect debugging process. International Journal of Industrial Engineering-Theory Applications and Practice 11(4): 396-405

Yamada S, Ohba M, Osaki S 1983 S-shaped reliability growth modelling for software error detection. IEEE. Trans. Rel. R-32: 475-478

Yamada S, Ohba M, Osaki S 1984 S-shaped software reliability growth models and their applications. IEEE. Trans. Rel. R-33: 289-291

Yamada S, Ohteria H, Narihisa H 1986 Software reliability growth models with testing-effort. IEEE. Trans. Rel. R-35: 19-23

Yamada S, Hishitani J, Osaki S 1993 Software reliability growth with a weibull test-effort: A model application. IEEE. Trans. Rel. R-42: 100-105

Zeephongsekul P, Xia G, Kumar S 1994 Software-reliability growth model: Primary failures generate secondary-faults under imperfect debugging. IEEE Trans. Rel. 43: 408-413 